\title{
Motion-Sensing Based Management System for Smart Context-Awareness Rehabilitation Healthcare
}

\author{
Tung-Hung Lu, Hsing-Chen Lin, Rong-Rong Chen, Ya-Ling Chen \\ Service Systems Technology Center, Industrial Technology Research Institute, Hsinchu, Taiwan \\ Email: tonylu@itri.org.tw \\ Received April 10, 2013; revised May 10, 2013; accepted May 17, 2013
}

Copyright (C) 2013 Tung-Hung Lu et al. This is an open access article distributed under the Creative Commons Attribution License, which permits unrestricted use, distribution, and reproduction in any medium, provided the original work is properly cited.

\begin{abstract}
In this paper, a motion-sensing based management system for smart context-awareness rehabilitation healthcare including various balance exercise is built by the integration of the physiological sensing and feedback coaching. The home-end system can not only provide the exercise coaching instruction, the balance stability analysis, and the motion similarity analysis in real-time, but also simultaneously transmit the user image, exercise skeleton streaming, center of pressure $(\mathrm{COP})$, center of gravity $(\mathrm{COG})$ and physiological information to the telecare-end center. According to the combination of the home-end and the telecare-end as well as the real-time care management of one-to-multiple personal balance exercise monitor, this system can provide user various personalized balance exercise prescription and cardiac rehabilitation coaching in an effectiveness rehabilitation exercise environment. Therefore, via this tele-system, the spinocerebellar ataxia (SCA) patients in balance rehabilitation stage not only can be monitored execution status of the rehabilitation exercise prescription, but also can be long-term monitored and evaluated the predicted goal of the rehabilitation exercise balance stability in order to improve patient's compliance.
\end{abstract}

Keywords: Motion-sensing Rehabilitation Exercise System; Care Service Platform; Balance Rehabilitation

\section{Introduction}

The cerebellum is a crucial part of the central nervous system, coordinating our movements, regulating the muscle tension, and controlling posture and gait. Various symptoms of uncoordinated movement and imbalance-indicative of spinocerebellar atrophy appear when pathological changes occur in the cerebellum. The official term for this disorder proposed by the Department of Health, Taiwan is spinocerebellar ataxia (SCA). Following onset, patients with SCA walk unsteadily (similar to a penguin); therefore, they are also termed "penguin family". SCA, which is among a group of genetic disorders, is primarily characterized by progressive incoordination of gait, and associated with poor coordination of hands, speech, and eye movements. One study indicated that Tai Chi helps improve the unsteady gait of patients with Parkinson's disease [1]. Hence, one of the hospitals in Taiwan attempted to improve the unsteady gait of SCA patients using medicine along with Tai Chi. Each week, volunteer professional instructors provided Tai Chi lessons to these patients at the hospital, allowing the patients to relieve emotional stress and simultaneously increase interaction between patients.
Based on this example, physical therapists may develop personalized home-based Balance Tai Chi rehabilitation exercise programs. By implementing Balance Tai Chi exercise prescriptions, patients are enabled to understand the effect that this exercise has on their physiological conditions and, with sufficient awareness, continue this exercise at home by themselves. Currently, tracking of patients prescribed with home-based balance exercises occurs weekly when they return to the hospital to practice the exercises. Thus, no objective and instant information is available as a basis for the hospital to evaluate the patients' balance status.

Under the rehabilitation model described previously, the existing traditional balance rehabilitation training must continue at the hospital under the supervision of a professional case manager. The rehabilitation exercises are constantly repeated, which become dull. Therefore, it is difficult for many patients to practice rehabilitation exercises according to the instructions of the Tai Chi coach at home, apart from problems regarding the correctness of the posture and the control of exercise duration and intensity. Patients are required to return to the hospital for rehabilitation follow-up once a week; however, transportation and caregivers' availability is another 
issue. In addition, the amount of time that the case managers are available may also be limited, because they can only assist $1-2$ patients concurrently. These factors affect the patients' willingness to continue and comply with their exercise prescriptions, often causing a deterioration of their symptoms that forces them to be re-admitted; consequently, medical resource utilization and burdens on the patients and their families increase [2].

Many existing studies have suggested introducing technology to improve patients' willingness to undergo rehabilitation. Videogames can be leveraged as a telehealth technology [3]. Telehealth technology is gaining attention as a promising strategy for acquiring accurate, reliable, and time-critical health marker data, reducing health care costs, empowering patients, and promoting disease self-management with resultant improved healthcare outcomes. Although telehealth technologies provide opportunities, several barriers relating to the acceptance and use by patient, caregiver, clinical support networks include problems in use of technology among older adults, lack of adequate training or support, lack of consensus on the value of the technology, and absence of adequate technology infrastructure [4].

To overcome these barriers, it will be important for designers of telehealth technologies to work closely with specific patients throughout the design and development process in order to learn how their capabilities relate to technology adoption and long-term use [3,5]. Videogames have already been proven to improve cognitive abilities, displaying a feasible alternative to more traditional aerobic exercise for middle-aged and older adults, and can be used to train stepping ability in older adults to reduce the risk of falls [6]. The use of Wii Sports and Wii Fit has been reported in an increasing number of studies involving people with motor deficits resulting from other causes such as balance and mobility in people with some diseases $[7,8]$.

Spinocerebellar ataxia (SCA) is a progressive, degenerative, genetic disease with multiple types. The signs and symptoms of an ataxia is one of a group of genetic disorders characterized by slowly progressive in coordination of gait and is often associated with poor coordination of hands, speech, and eye movements. With progression of the disease, patients lose postural stability and have gait dysfunction, difficulty in managing activities of daily living, and frequent falls. Although some motor dysfunctions may be alleviated with drug therapy, characteristics such as postural instability are less responsive to medication and require alternative approaches. The relevance of this study is timely; technology developments within the videogame sector are continually enhancing and pushing the boundaries to bring innovative and exciting modes of interaction to consumers. With this knowledge, maximizing the utility of such technologies into health services can offer methods for delivery of rehabilitation and training services.

In this paper, a motion-sensing based management system for smart context-awareness rehabilitation care was developed, consisting of a home-end motion-sensing rehabilitation exercise system and a remote care service platform. The home-end motion-sensing rehabilitation exercise system is equipped with various balance rehabilitation exercise models. The users' whole-body image is captured using a motion-sensing camera and projected into virtual exercise scenarios. The users' compliance is computed instantly through a motion analysis technology to provide exercise coaching instructions. The balance status of the users during exercise is subject to a realtime cross-analysis: the center of gravity (COG) trace is calculated using a depth image captured by a motionsensing camera; and the center of pressure (COP) trace is calculated using a pressure array sensor. In addition, the physiological information is incorporated to analyze physiological changes before, during, and after exercise. Through VoIP and streaming data integration technology, this system provides a real-time, one-to-multiple remote care service platform for exercise monitoring and interactive instructions.

\section{System Framework}

Figure 1 shows the motion-sensing based management system for smart context-awareness rehabilitation care, which is a home-end interactive motion-sensing rehabilitation exercise system that assists users in their balance rehabilitation exercises using several real-time sensors (motion-sensing camera, webcam, biomedical sensor, and pressure array sensor) through the wire/wireless connection (i.e., similar to the internet of things (IoT) model for physiological monitoring using motion-sensing rehabilitation). This system allows users to maintain their optimum exercise tolerance benchmark (that they reached in the hospital) within a certain range, and to enhance their compliance with the rehabilitation exercise and their balance function. Through physiological data that are detected automatically by a wireless physiological measuring device, this system au-to-computes the risks of patients encountering danger when exercising by themselves. Through a motion-sensing camera (e.g., Microsoft Kinect or ASUS Xtion PRO), the correctness of the users' movements is automatically analyzed. Their balance status is cross-analyzed using a pressure array sensor and a motion-sensing camera. The remote care service platform integrates the VoIP, motion image analysis, exercise skeleton streaming, balance analysis, and physiological information convergence technologies to construct a personal health management platform server, enabling care managers to simultaneously and remotely supervise the actual conditions and physiological changes of several users during exercise. This system automati- 
cally alerts the telecare managers when the users' physiological measurement or balance data are abnormal or when the system calculated risk is higher than usual. By using the data transmitted by the rehabilitation exercise system, the care managers can understand the problem and address it through VoIP or real-time video-conferencing to the patient's home. In the following section, we introduce the home-end motion-sensing rehabilitation exercise system, the remote care service platform, and the clinical decision support system (CDSS) of the motionsensing based management system for smart contextawareness rehabilitation care.

\subsection{Motion-Sensing Rehabilitation Exercise System}

The framework of the motion-sensing rehabilitation ex- ercise system, as shown in Figure 2, consists of an exercise template database and modules for motion feature analysis, rehabilitation coaching, vital sign fusion, balance analysis, care service platform interface, and backend rehabilitation CDSS. The various balance re-habilitation exercises, which were designed by physicians and led in the system by a virtual exercise coach, are recorded through a professional motion-capture system and stored in the exercise template database, allowing users to execute rehabilitation exercises by following the virtual coach's instructions. If user movements do not meet the system standards, the skeleton motion trace of the users is captured by the motion-sensing camera and the motion feature analysis module. Movements and gestures of the users can then be interpreted by comparing them instantly with the standard skeleton exercise trace of the

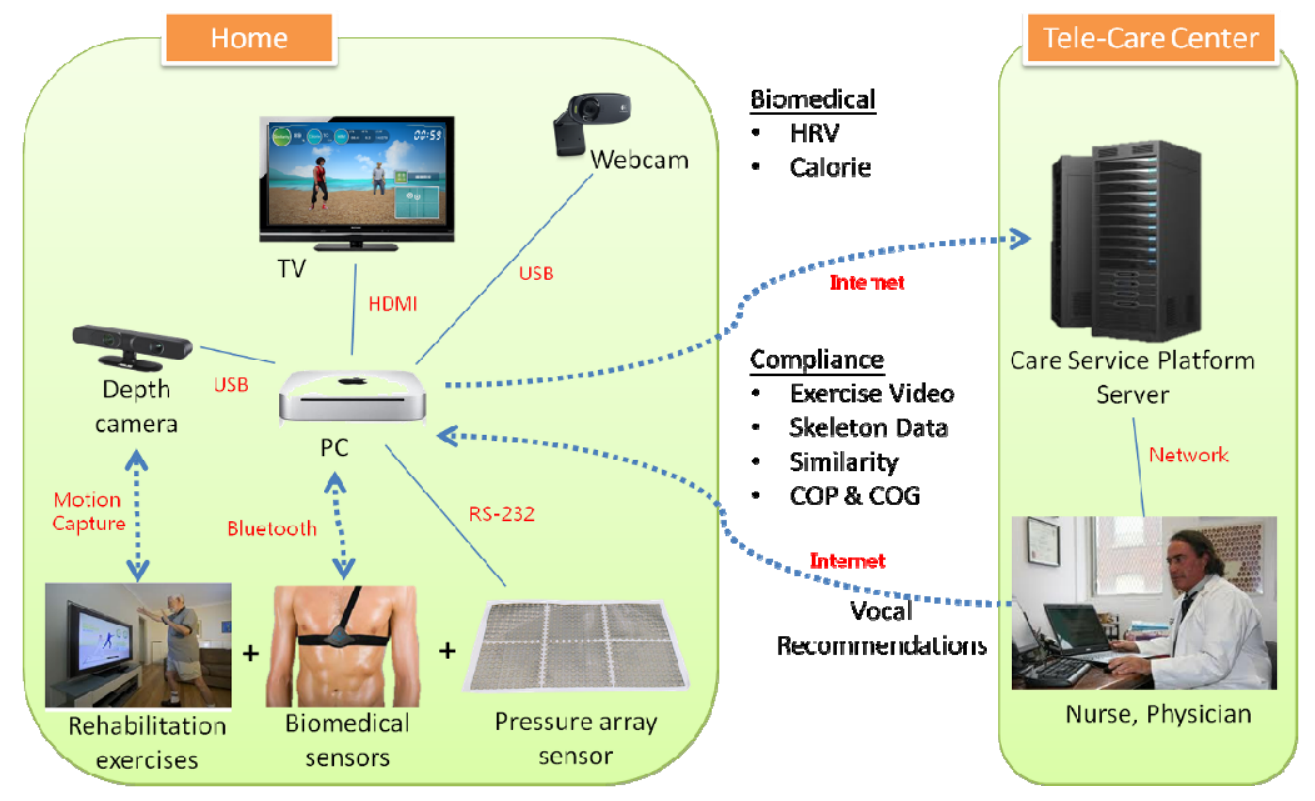

Figure 1. System framework.

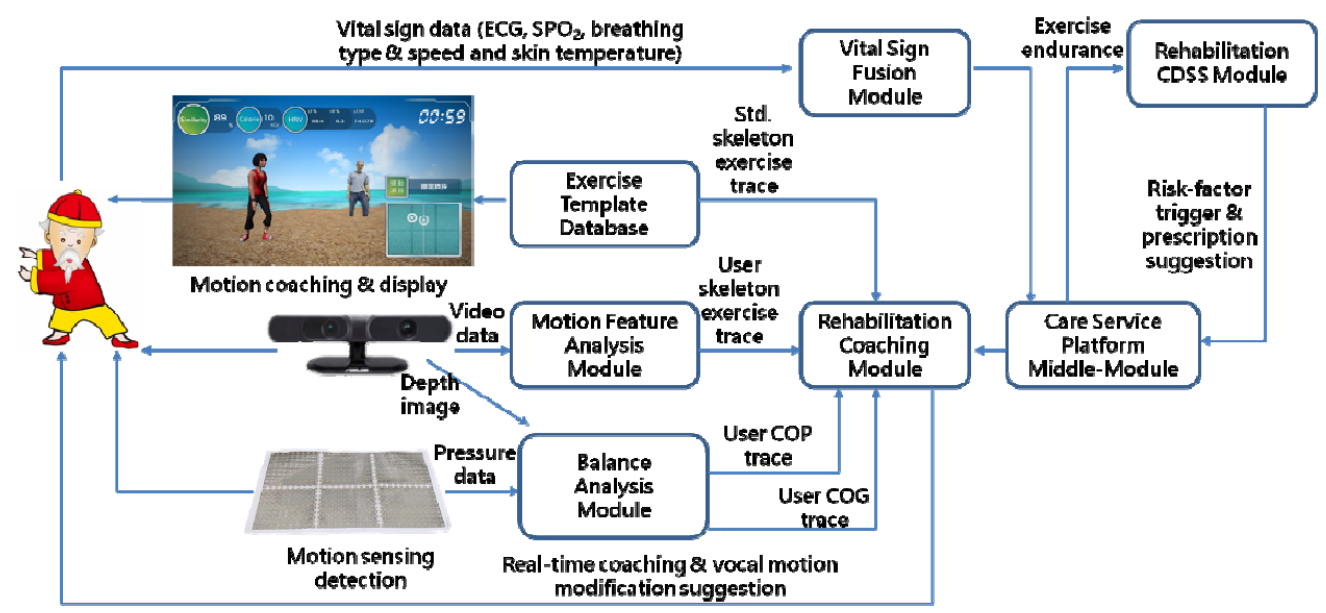

Figure 2. Framework of the motion-sensing rehabilitation exercise system. 
exercise template database. In addition, the balance status of the users during exercise can be analyzed instantly through the depth image of the users captured by a motion-sensing camera, the foot pressure measured by a pressure array sensor, and the COG and COP computed by the balance analysis module. The system provides an instant voice feedback to correct the users' movements through statistics of balance analysis and motion similarity comparison received by the rehabilitation coaching module. With an appropriate wireless physiological measureing device and a sensor, information about the physiological status during exercise can be collected and monitored using a vital sign collection module. The back-end rehabilitation CDSS module and the interfaced personal care service platform module are immediately triggered if an abnormal balance or physiological status of the users is detected. The system then automatically alerts the telecare case managers to ensure the users' exercise safety.

In addition, this system provides virtual game scenarios, as shown in Figure 3, which incorporates the users' RGB image into virtual scenarios; it also allows for exercising with a virtual coach to promote the patients' enjoyment for rehabilitation. In addition, the system employs sensing devices and a 3D motion-sensing camera to accurately understand the users' exercise progress, including calories burned, heart rate variability (HRV; including $\mathrm{LF} \%, \mathrm{HF} \%$, and $\mathrm{LF} / \mathrm{HF}$ ), real-time $\mathrm{COP}$, and exercise compliance represented by motion similarity $[2$, 9-12].

\subsection{Care Service Platform}

Through integration of the home-end motion-sensing rehabilitation exercise system, various sensing devices, and VoIP, the remote personal care service platform is a back-end monitoring platform for caregivers, allowing simultaneous 30 monitoring online users practicing different rehabilitation exercises using the motion-sensing rehabilitation exercise system. The care managers can supervise the exercise conditions of six users simultaneously. Through both the physiological data and COP, which are respectively detected by a wireless physiological measuring device and a pressure array sensor, this system auto-computes the exercise risk of the patients.

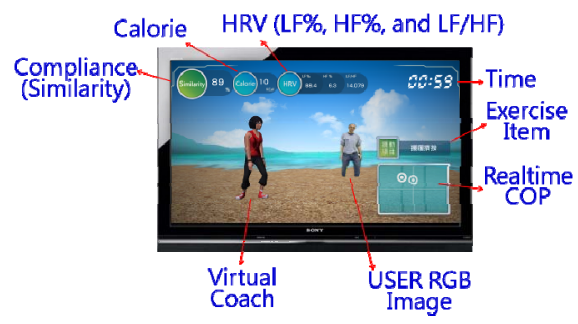

Figure 3. The virtual game interface for motion-sensing rehabilitation exercise scenarios.
The system automatically alerts the telecare case managers when the physiological measurement is abnormal or when the system calculated risk is higher than usual. Using the data received by the rehabilitation exercise system, the case managers can understand and address the problem through VoIP or real-time videoconferencing to the patients' home.

The real-time exercise information that is transmitted to the care service platform from the home-end motionsensing rehabilitation exercise system consists of physiological information (i.e., heart rate, exercise intensity, and HRV) and monitored exercise information (i.e., motion similarity, skeleton node, COP, and COG). Specifically, 60 data entries per second are received from the electrocardiogram, with a data rate of 1204 bps. Fifteen nodes are allocated to a skeleton, each consisting of an $\mathrm{X}$-axis, a $y$-axis, and a z-axis. Thirty entries of skeleton data are obtained per second, with approximately $1 \mathrm{~K}$ data received per second. Compliance is denoted by an integer representing the percentage of motion similarity.

The framework of the care service platform, as shown in Figure 4, consists of application, computing, repository, and integration service layers. Through the interface between the Super Socket series integrated service module and the home-end motion-sensing rehabilitation exercise system, the care service platform receives a variety of physiological data and exercise monitoring information from the home-end system. The platform then instantly computes and statistically analyzes the data transmission frequency (times/second), the data quantity (total bytes), and the transmission quantity (bytes/s) through the skeleton, HRV, heart rate, exercise intensity, COP, and COG processing modules in the computing services. The received raw data and the computed results obtained through the computing services can be stored in the repository services to provide the case managers with anytime access to the users' exercise history. The application services, comprising the videoconferencing, exercise instruction, compliance display, and motion and vital sign display modules, can display the exercise conditions of several online users on the monitor interface, as shown in Figure 5 (including skeleton streaming, an audio/visual interface for videoconferencing, and the vital signs of multiple online users). The case managers can immediately understand and address issues through videoconferencing if the users' compliance declines, as shown in Figure 6.

\subsection{Clinical Decision Support System}

The medical profession has developed from general practice in the past to specialty-based practice in the present. Currently, the development of each specialty emphasizes further specialization. Therefore, when treating patients who require cross-disciplinary integrated care, 


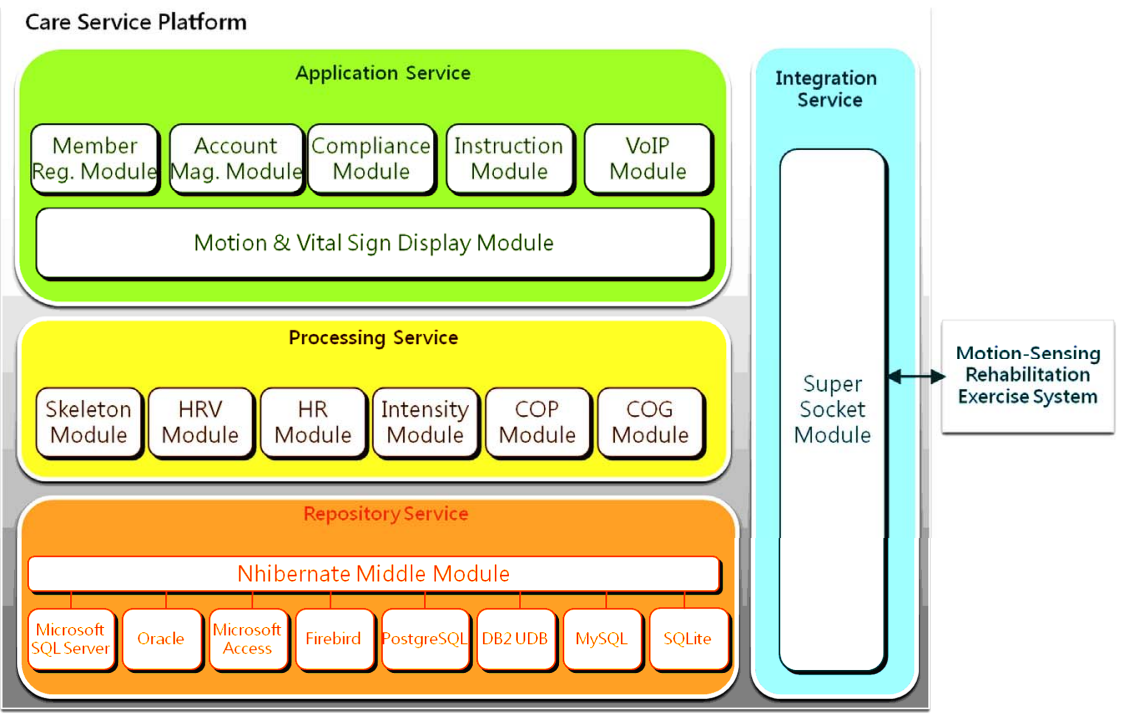

Figure 4. Framework of the care service platform.

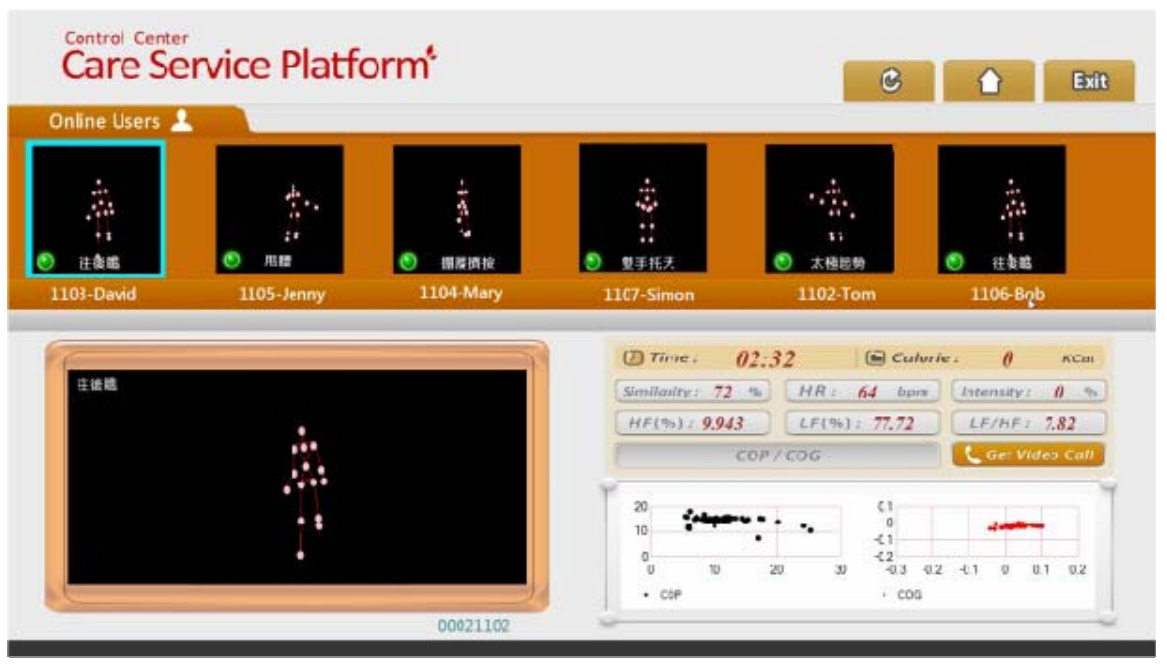

Figure 5. The exercise monitoring interface for six patients.

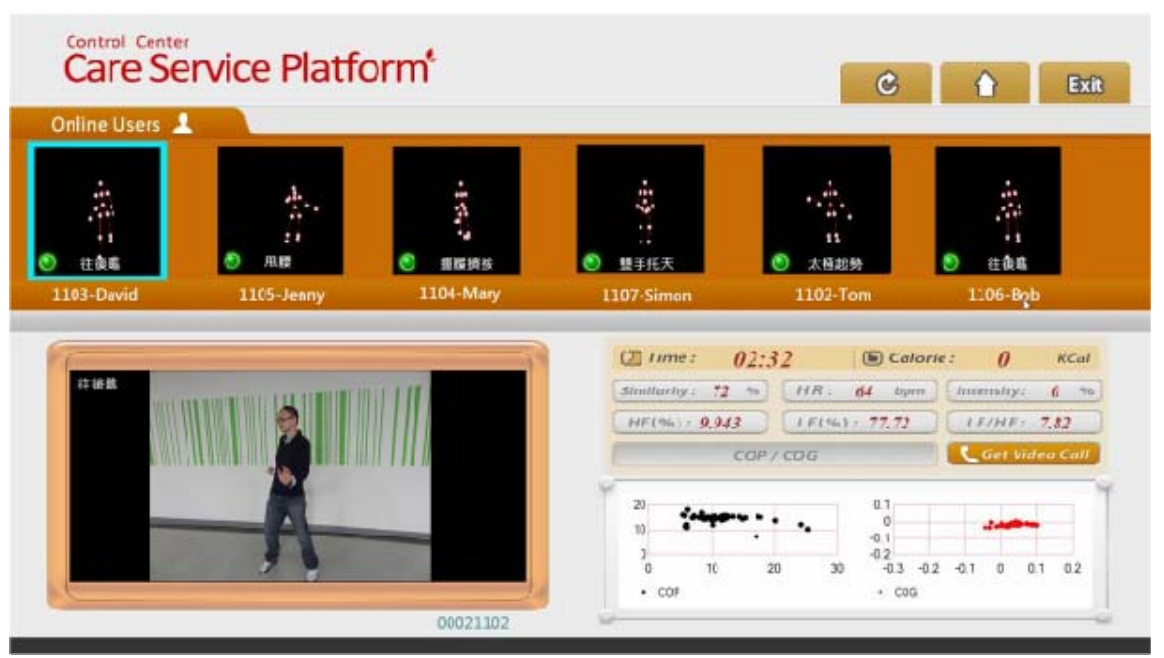

Figure 6. The audio/visual interface for videoconferencing. 
specialized physicians are often unable to fully address patients' complaint, resulting in time-consuming and repeated visits. The CDSS can simultaneously provide professional cross-disciplinary decision support for medical personnel, solve the problem of insufficient interdisciplinary knowledge, enhance diagnosis and service performance, and lower the professional threshold for providing rehabilitation care to patients with SCA.

The framework of the CDSS, as shown in Figure 7, is designed based on the execution engine of a core clinical pathway, providing different user interfaces for relevant medical personnel to access various decision support information. Through a back-end management function, medical experts can also maintain information related to multiple clinical pathways and rules to adjust existing information regarding contexts applicable to various clinical decisions. The CDSS, comprising a process control engine and professional medical knowledge rules, allows medical personnel to better understand the patients' status and provide personalized care services.

\section{Pilot Verification}

This motion-sensing based management system for smart context-awareness rehabilitation healthcare now is being tested in clinical settings of one hospital in Taiwan. The conditions of the test target users group are: ages from 20 to 80 years old; diagnosis of SCA confirmation; gait $\leq 7$ or stance $\leq 5$ in the scale for the assessment and rating of ataxia (SARA scale).

These group exercise testing at hospital once a week for five weeks, supervised by lots of the motion-sensing based management systems for smart context-awareness rehabilitation healthcare. Participants will be instructed by virtual coach to maintain their usual exercise via motion-sensing based Balance Tai Chi rehabilitation exercise program. COP and COG matching are measured by pressure array sensor during the intervention mainly for assessment standing balance data (e.g., center shift degree in $\mathrm{x}-\mathrm{y}$-axis and center shift trace distance etc). In

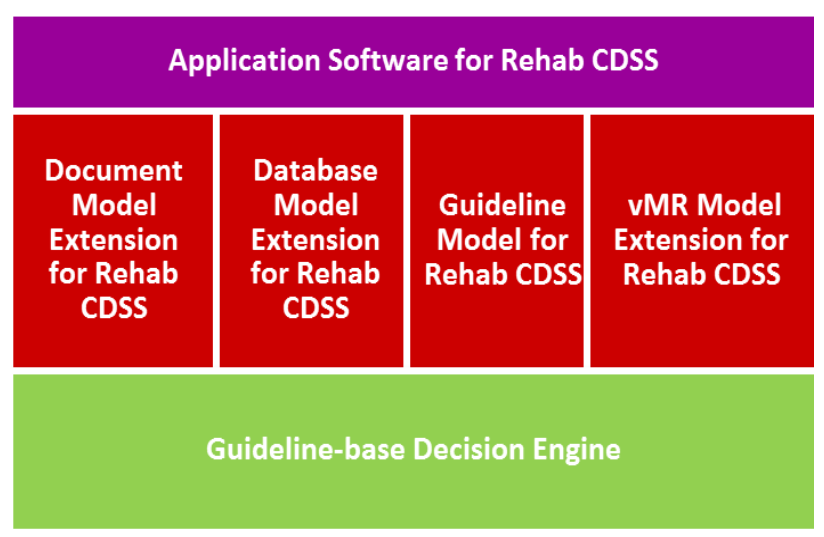

Figure 7. Framework of the clinical decision support system. addition, motion compliance (or motion similarity) is one of the key indicators to analyze the patients motion accuracies compared with virtual coach. Case manager in front of care service platform also can monitor the rehabilitation status via this back-end system for patients safe exercise in terms of rehabilitation CDSS. Therefore, pilot verification can evaluate the balance status of the patients with SCA via this motion-sensing based management systems for smart context-awareness rehabilitation healthcare in terms of the COP, COG matching, and motion similarity.

\section{Conclusion}

A system was designed to develop a set of balance rehabilitation exercises for patients with SCA for continuous and safe practice at home through information and communication technology. By integrating physiological monitoring and feedback coaching with a telecare center that enables real-time one-to-multiple personal exercise monitoring by case managers, this home-end rehabilitation exercise system provides users with diverse personalized balance exercise prescriptions and rehabilitation exercise coaching in an effective home exercise environment. Consequently, patients are enabled to maintain their optimum exercise tolerance benchmark that they achieved in the hospital.

\section{REFERENCES}

[1] F. Li, P. Harmer, K. Fitzgerald, E. Eckstrom, R. Stock, J. Galver, G. Maddalozzo and S. S. Batya, "Tai Chi and Postural Stability in Patients with Parkinson's Disease," New England Journal of Medicine, Vol. 366, No. 6, 2012, pp. 511-519. doi:10.1056/NEJMoa1107911

[2] T. H. Lu, H. C. Lin, Y. H. Lee, R. R. Chen, H. L. Chen, S. Y. Chang, J. D. Chen, B. R. Wu and T. H. Wu, "A Motion-Sensing Enabled Personalized Exercise System for Cardiac Rehabilitation," IEEE 14th International Conference on e-Health Networking, Applications and Services, Beijing, 10-13 October 2012, pp. 167-171.

[3] H. R. Marston and S. T. Smith, "Interactive Videogame Technologies to Support Independence in the Elderly: A Narrative Review," Game for Health, Vol. 1, No. 2, 2012, pp. 139-152. doi:10.1089/g4h.2011.0008

[4] S. Mattke, L. Klautzer, T. Mengistu, J. Garnett, J. Hu and $\mathrm{H}$. Wu, "Health and Well-Being in the Home: A Global Analysis of Needs, Expectations, and Priorities for Home Health Care Technology," RAND Corporation, Santa Monica, 2010.

[5] G. Demris, N. Charness, E. Krupinski, D. Ben-Arieh, K. Washington, J. Wu and B. Farberow, "The Role of Human Factors in Telehealth," Telemedicine and E-Health, Vol. 16, No. 4, 2010, pp. 446-453. doi:10.1089/tmj.2009.0114

[6] B. Guderian, L. A. Borreson and L. E. Sletten, "The Cardiovascular and Metabolic Responses to Wii Fit Video 
Game Playing in Middle-Aged and Older Adults," Journal of Sport Medicine and Physical Fitness, Vol. 50, No. 4, 2010, pp. 436-442.

[7] J. E. Deutsch, D. Robbins, J. Morrison and P. G. Bowlby, "Wii-Based Compared to Standard of Care Balance and Mobility Rehabilitation for Two Individuals Post-Stroke," Virtual Rehabilitation International Conference, Deutsch, Haifa, 2 June 2009, pp. 117-120.

[8] E. Bainbridge, S. Bevans, B. Keeley and K. Oriel, "The Effects of the Nintendo Wii Fit on Community-Dwelling Older Adults with Perceived Balance Deficits: A Pilot Study," Physical \& Occupational Therapy in Geriatrics, Vol. 29, No. 2, 2011, pp. 126-135. doi:10.3109/02703181.2011.569053

[9] G. Burdea, "Virtual Rehabilitation-Benefits and Challenges," International Medical Informatics Association
Yearbook of Medical Informatics, International Medical Informatics Association, Geneva, 2003.

[10] R. A. Clark, Y. H. Pua, K. Fortin, C. Ritchie, K. E. Webster, L. Denehy and A. L. Bryant, "Validity of the Microsoft Kinect for Assessment of Postural Control," Gait Posture, Vol. 36, No. 3, 2012, pp. 372-377. doi:10.1016/j.gaitpost.2012.03.033

[11] A. Ramchandani, K. Carroll, R. Buenaventura, J. Douglas and J. Liu, "Wii-Habilitation Increases Participation in Therapy," IEEE Conference of Virtual Rehabilitation, Vancouver, 25-27 August 2008, p. 69.

[12] C. Watters, S. Oore, M. Shepherd, A. Abouzied, A. Cox, M. Kellar, H. Kharrazi, F. Liu and A. Otley, "Extending the Use of Games in Health Care," 39th Annual Hawaii International Conference on System Sciences, Kauai, Hawaii, 4-7 January 2006. 